ARTICLE

\title{
Petahertz non-linear current in a centrosymmetric organic superconductor
}

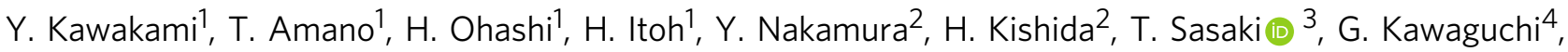 \\ H. M. Yamamoto (iD ${ }^{4}$, K. Yamamoto ${ }^{5}$, S. Ishihara ${ }^{1}$, K. Yonemitsu ${ }^{6} \&$ S. Iwai (i) ${ }^{1 凶}$
}

Charge acceleration during an intense light field application to solids attracts much attention as elementary processes in high-harmonic generation and photoelectron emission. For manipulating such attosecond dynamics of charge, carrier-envelope-phase (CEP: relative phase between carrier oscillation of light field and its envelope function) control has been employed in insulators, nanometal and graphene. In superconducting materials, collective control of charge motion is expected because of its strongly coherent nature of quasiparticles. Here we report that, in a layered organic superconductor, a non-linear petahertz current driven by a single-cycle 6 femtosecond near infrared field shows up as second harmonic generation (SHG), which is in contrast to the common belief that even harmonics are forbidden in the centrosymmetric system. The SHG represents a CEP sensitive nature and an enhancement near the superconducting temperature. The result and its quantum manybody analysis indicate that a polarized current is induced by non-linear acceleration of charge, which is amplified by superconducting fluctuations. This will lead to petahertz functions of superconductors and of strongly correlated systems.

\footnotetext{
${ }^{1}$ Department of Physics, Tohoku University, Sendai 980-8578, Japan. ${ }^{2}$ Department of Applied Physics, Nagoya University, Nagoya 464-8603, Japan.

${ }^{3}$ Institute for Materials Research, Tohoku University, Sendai 980-8577, Japan. ${ }^{4}$ Institute for Molecular Science, Okazaki 444-8585, Japan. ${ }^{5}$ Department of Applied Physics, Okayama University of Science, Okayama 700-0005, Japan. ${ }^{6}$ Department of Physics, Chuo University, Tokyo 112-8551, Japan.

凶email: s-iwai@tohoku.ac.jp
} 
A n electromagnetic oscillation of light cannot directly drive a polarized current because of its symmetric nature on the time axis (i.e., the time average of the oscillation is zero). However, recent developments of ultrashort laser technologies enable us to control the direction of charge motion by carrierenvelope phase (CEP) control of a strong light field ${ }^{1-12}$. Considering non-linear light-matter interactions during an ultrashort pulse, we can expect petahertz control of the polarized current in superconducting materials.

In the non-linear regime (where a non-linear electric displacement along the polarization axis $D(t)$ is induced by an electric field), a net current given by $j(t) \propto \int_{0}^{t} D(\tau) \mathrm{d} \tau$ does not necessarily vanish for a few-cycle or single-cycle field. Interestingly, such a non-linear current can be modulated by the CEP as shown in Fig. 1b, if the CEP of $E(t)=E_{0}(t) \sin \left(\omega t-\varphi_{\text {CEP }}\right)\left(E_{0}\right.$ $(t)$ : envelope function of a single-cycle pulse, $\varphi_{\mathrm{CEP}}$ : CEP) is preserved in $D(t) \propto \sin \left(\omega t-\varphi_{\text {CEP }}\right)$. This is in contrast to the fact that a net current driven by a light-field is zero in the linear regime. Such a non-linear current can break the symmetry of the charge density in momentum space (or equivalently a breaking of the spatial inversion symmetry in the sense that the induced current is no longer described as an odd function of $E(t)$ of the moment), resulting in current-induced second harmonic generation $(\mathrm{SHG})^{13-16}$. Thus, the SHG (and the spatial inversion symmetry breaking) is induced even in the centrosymmetric system, if a non-linear current is driven by light.

Our target material is a layered organic superconductor $\kappa$ $(\mathrm{BEDT}-\mathrm{TTF})_{2} \mathrm{Cu}\left[\mathrm{N}(\mathrm{CN})_{2}\right] \mathrm{Br}^{17-21}$ with a transition temperature $T_{\mathrm{SC}}=11.6 \mathrm{~K}$ (Fig. 1c). Superconducting fluctuations above $T_{\mathrm{SC}}$ $\left(T_{\mathrm{SC}}<T<\sim 2 T_{\mathrm{SC}}\right)$ have been discussed in analogy with a pseudogap in high- $T_{\mathrm{SC}}$ superconducting cuprates in the temperature$t / U_{\text {dimer }}$ phase diagram ${ }^{20,21}$, where $t / U_{\text {dimer }}$ is the ratio of an interdimer transfer integral $t$ to the effective on-site Coulomb energy for a dimer $U_{\text {dimer, }}$ as shown in Fig. 1c. In superconductors, optical responses have been discussed in terms of nonequilibrium quasi-particle dynamics on the time scale of picosecond $^{22-25}$, coherent excitation of the Higgs mode ${ }^{26}$ and lightinduced superconductivity ${ }^{27,28}$. On the other hand, petahertz light functions, driven by the non-linear current during a light pulse is also expected to be characteristic, reflecting the ultrafast time scale of superconducting fluctuations in $\kappa$-(BEDT-TTF $)_{2} \mathrm{Cu}$ $\left[\mathrm{N}(\mathrm{CN})_{2}\right] \mathrm{Br}^{29}$.
In this article, we report SHG for a strong light field (16 MV/ $\mathrm{cm}=1.6 \mathrm{~V} / \mathrm{nm}$ ) in a centrosymmetric organic superconductor $\mathrm{\kappa}$ (BEDT-TTF $)_{2} \mathrm{Cu}\left[\mathrm{N}(\mathrm{CN})_{2}\right] \mathrm{Br}$ (single crystal). A CEP dependence of the SHG shows that it is induced by the non-linear current. Superconducting fluctuations above $T_{\mathrm{SC}}$ amplify the SHG.

\section{Results}

SHG and its increase near superconducting temperature. Figure 2a shows spectra (measured at $6 \mathrm{~K}$ ) of SHG and third harmonic generation (THG) with peak energies of $1.5 \mathrm{eV}$ [SHG, red $\left(\mathbf{E}_{\mathrm{Fund}}|| \mathbf{c}, \mathbf{E}_{\mathrm{SH}}|| \mathbf{c}\right)$ and green $\left(\mathbf{E}_{\text {Fund }}|| \mathbf{a}, \mathbf{E}_{\mathrm{SH}}|| \mathbf{c}, \times 0.73\right)$ lines] and $2.2 \mathrm{eV}$ [THG, blue line $\left.\left(\mathbf{E}_{\text {Fund }}|| \mathbf{c}, \mathbf{E}_{\mathrm{SH}}|| \mathbf{c}, \times 0.024\right)\right]$ for the fundamental photon energy of $0.75 \mathrm{eV}$. Here, $\mathbf{E}_{\mathrm{Fund}}, \mathbf{E}_{\mathrm{SH}}$, and $\mathbf{E}_{\mathrm{TH}}$ indicate the electric fields of the fundamental light, SHG and THG, respectively. Note that the SHG is originally forbidden in this centrosymmetric system (orthorhombic with Pnma symmetry ${ }^{30,31}$ ) in a conventional perturbation theory. However, the SHG is observed, and it is ca. 1/50 times as intensive as the THG.

As shown in the upper panel of Fig. 2b, the SHG is polarized parallel to the $\mathbf{c}$-axis $\left(\mathbf{E}_{S \mathrm{H}}|| \mathbf{c}\right)$ for both excitation polarizations $\left[\mathbf{E}_{\text {Fund }}|| \mathbf{c}\right.$ (red line), $\mathbf{E}_{\text {Fund }}|| \mathbf{a}$ (green line)], although the THG shows the usual polarization which is the same as that of the fundamental pulse (lower panel). Such unusual polarization dependence of the SHG is discussed below. We performed SHG and THG measurements with an incident angle of smaller than three-degree to minimize surface $\mathrm{SHG}^{32}$. In fact, the results above $\left(\mathbf{E}_{\mathrm{Fund}}|| \mathbf{c}, \mathbf{E}_{\mathrm{SH}}|| \mathbf{c}\right)$ and $\left(\mathbf{E}_{\mathrm{Fund}}|| \mathbf{a}, \mathbf{E}_{\mathrm{SH}}|| \mathbf{c}\right)$ are confirmed by rotating the sample by $90^{\circ}$ not to depend on the s-polarized or $p$ polarized configuration under such a restricted condition, although the $s$-polarized or $p$-polarized configuration is indistinguishable for the incident angle of $0^{\circ}$. Moreover, the dependences on the CEP and the temperature (which are shown below) are incompatible with SHG induced by static geometries such as a surface or a point defect. Thus, the observed SHG is not attributed to surface SHG. The possibility of symmetry breaking induced by photo-carrier generation near the surface is also excluded by the fact that the lifetime of carriers (ca. $500 \mathrm{fs})^{29}$ (Supplementary note 3 ) is much longer than the time scale of the non-linear effect which drives the SHG (ca. $40 \mathrm{fs}$ is evaluated from the bandwidth as discussed later).

Figure $2 \mathrm{c}$ shows the temperature dependence of the peak intensities for SHG $\left[I_{\mathrm{SH}}\right.$ : closed red circles $\left(\mathbf{E}_{\mathrm{fund}}|| \mathbf{a}, \mathbf{E}_{\mathrm{SH}}|| \mathbf{c}\right)$,
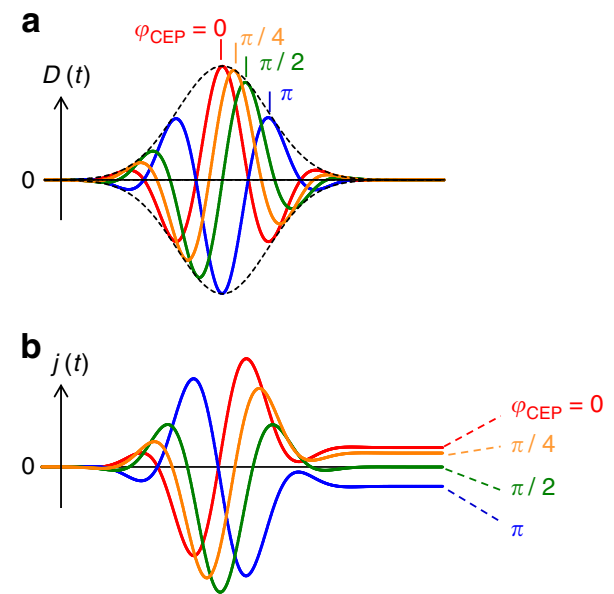

C

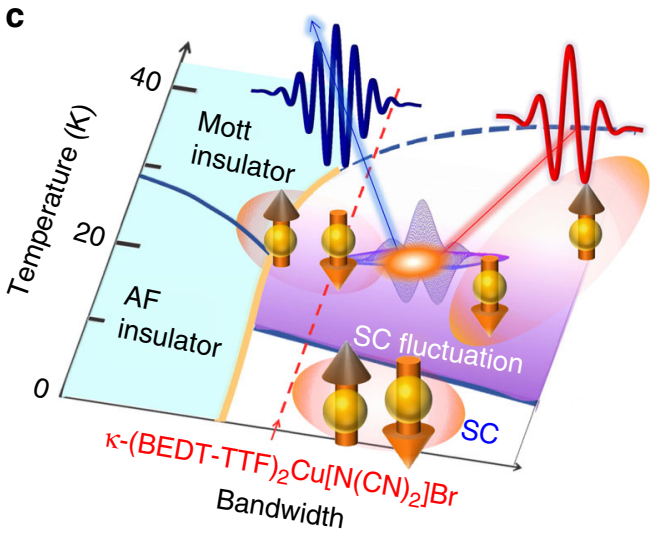

Fig. 1 CEP control of non-linear current and phase diagram of $\kappa$-type BEDT-TTF salts. a Non-linear electric displacement $D(t)$ induced by electric field $E(t)=E_{0}(t) \sin \left(\omega t-\varphi_{\text {CEP }}\right)$, where $\varphi_{\text {CEP }}=0$ (red), $1 / 4 \pi$ (orange), $1 / 2 \pi$ (green), $\pi$ (blue) $\left(E_{0}(t)\right.$ : envelope of a single-cycle pulse) (we assume $\left.D(t) \propto \sin \left(\omega t-\varphi_{\mathrm{CEP}}\right)\right]$. b Non-linear light-induced current $j(t) \propto \int D(\tau) \mathrm{d} \tau$. c Temperature- $t / U_{\text {dimer }}$ (band width) phase diagram of $\kappa-(B E D T-T T F)_{2} X$, which is extracted based on controlling the chemical pressure $\left(=t / U_{\text {dimer }}\right)$. 
a

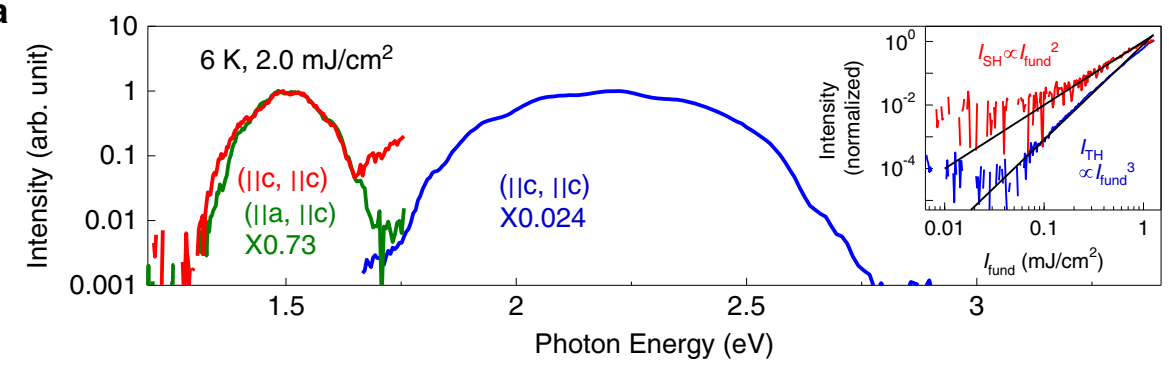

b

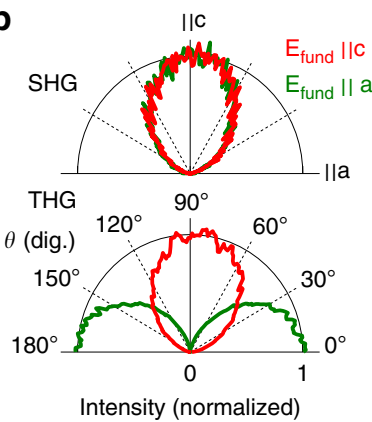

C

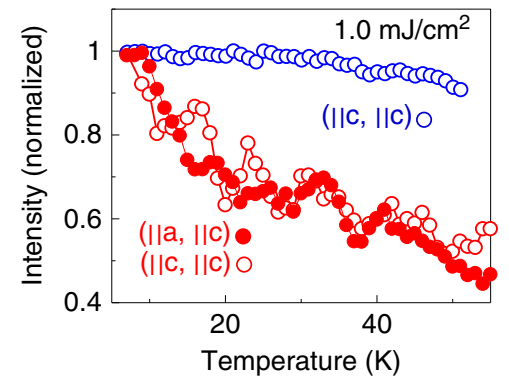

d

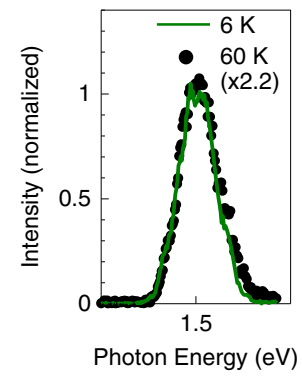

Fig. 2 SHG and THG spectra of $\kappa-(B E D T-T T F) 2 C u[N(C N) 2] B r$, and their polarization and temperature dependences. a The red and green lines indicate the SHG spectra of single crystalline $\kappa-(\mathrm{BEDT}-\mathrm{TTF})_{2} \mathrm{Cu}\left[\mathrm{N}(\mathrm{CN})_{2}\right] \mathrm{Br}(6 \mathrm{~K})$ for the polarizations of $\left(\mathbf{E}_{\text {fund }}|| \mathbf{c}, \mathbf{E}_{\mathrm{SH}}|| \mathbf{c}\right)\left(\right.$ red line) and $\left(\mathbf{E}_{\mathrm{fund}}|| \mathbf{a}, \mathbf{E}_{\mathrm{SH}}|| \mathbf{c}\right)$ (green line, $\times 0.73)$. The blue line shows the THG spectrum $(\times 0.024)$ for $\left(\mathbf{E}_{\text {fund }}|| \mathbf{c}, \mathbf{E}_{\mathrm{TH}}|| \mathbf{c}\right)$. The intensity of the fundamental light $\left(I_{\text {fund }}=E_{\text {fund }}{ }^{2}\right)$ is $2 \mathrm{~mJ} /$ $\mathrm{cm}^{2}$. Inset shows $I_{\text {fund }}$ dependences of $I_{\mathrm{SH}}$ (red line for $\mathbf{E}_{\text {fund }}|| \mathbf{c}, \mathbf{E}_{\mathrm{SH}}|| \mathbf{c}$ ) and $I_{\mathrm{TH}}$ (blue line for $\mathbf{E}_{\text {fund }}|| \mathbf{c}, \mathbf{E}_{\mathrm{TH}}|| \mathbf{c}$ ). b Polarization dependences of $I_{\mathrm{SH}}$ (upper panel) and $I_{\mathrm{TH}}$ (lower panel) for $\mathbf{E}_{\mathrm{fund}}|| \mathbf{c}$ (red line) and $\mathbf{E}_{\mathrm{fund}}|| \mathbf{a}$ (green line), respectively. $\mathbf{c}$ Temperature dependences of $I_{\mathrm{SH}}$ (closed red circles: $\mathbf{E}_{\mathrm{fund}}|| \mathbf{a}$, $\mathbf{E}_{\mathrm{SH}}|| \mathbf{c}$, open red circles: $\mathbf{E}_{\text {fund }}|| \mathbf{c}, \mathbf{E}_{\mathrm{SH}}|| \mathbf{c}$ ) and $I_{\mathrm{TH}}$ (open blue circles, $\mathbf{E}_{\mathrm{fund}}|| \mathbf{c}, \mathbf{E}_{\mathrm{TH}}|| \mathbf{c}$ ). Both are normalized by the respective intensities at $6 \mathrm{~K}$. $\mathbf{d} \mathrm{SHG}$ spectra ( $\mathbf{E}_{\text {fund }}|| \mathbf{a}, \mathbf{E}_{\mathrm{SH}}|| \mathbf{c}$ ) at $6 \mathrm{~K}$ (green line) and at $60 \mathrm{~K}$ (black dots).

open red circles ( $\left.\left.\mathbf{E}_{\text {fund }}|| \mathbf{c}, \mathbf{E}_{\mathrm{SH}}|| \mathbf{c}\right)\right]$ and THG ( $I_{\mathrm{TH}}$ : blue) (normalized by the intensities at $6 \mathrm{~K}$ ), indicating that the SHG grows up toward $T_{\mathrm{SC}}$ from high temperature, although $I_{\mathrm{TH}}$ does not depend on the temperature. The fluctuation of $I_{\mathrm{SH}}$ for $\left(\mathbf{E}_{\mathrm{fund}} \mid\right.$ $\left.\left|\mathbf{c}, \mathbf{E}_{\mathrm{SH}}\right| \mid \mathbf{c}\right)$ is larger than that for $\left(\mathbf{E}_{\text {fund }}|| \mathbf{a}, \mathbf{E}_{\mathrm{SH}}|| \mathbf{c}\right)$ because of stray lights from the intense/ broad THG. The spectral shape is almost independent of the temperature in the range between 6 and $60 \mathrm{~K}$ as shown in Fig. 2d, showing that the net intensity of the SHG is increased near $T_{\mathrm{SC}}$. Such temperature dependence of the SHG, which cannot be understood by a conventional perturbation theory, clearly shows that the observed SHG is related to the superconducting fluctuations ${ }^{20,21}$.

CEP dependence of SHG. Considering that the pulse width of 6 fs is shorter than the lower limit of the electron-electron scattering time [ca. $40 \mathrm{fs}=h /$ (inter-dimer transfer integral)] where the transfer integral is about $0.1 \mathrm{eV}$ in organic super conductors, a possible mechanism for the unconventional SHG is the nonlinear current discussed above. Furthermore, the temperature dependence (Fig. 2c) shows that the non-linear current is enhanced by the superconducting fluctuations.

We can demonstrate relevance of this scenario by measuring the CEP dependence of the SHG, because the non-linear current is sensitive to the CEP as mentioned above (Fig. 1b). Figure $3 \mathrm{~d}$ shows the intensity of the SHG as a function of the relative CEP $\left(\Delta \varphi_{\mathrm{CEP}}\right)$. During a period of the CEP, the SHG shows two maxima at around $\Delta \varphi_{\mathrm{CEP}}=1 / 2 \pi$ and $3 / 2 \pi$. Considering that the directions of the light-induced current cannot be distinguished by the SHG measurement, i.e., that currents with a same amplitude and opposite directions give same SHG intensities, the above $\Delta \varphi_{\text {CEP }}$ dependence is quite reasonable. This CEP dependence of the SHG is completely consistent with the fact that the a
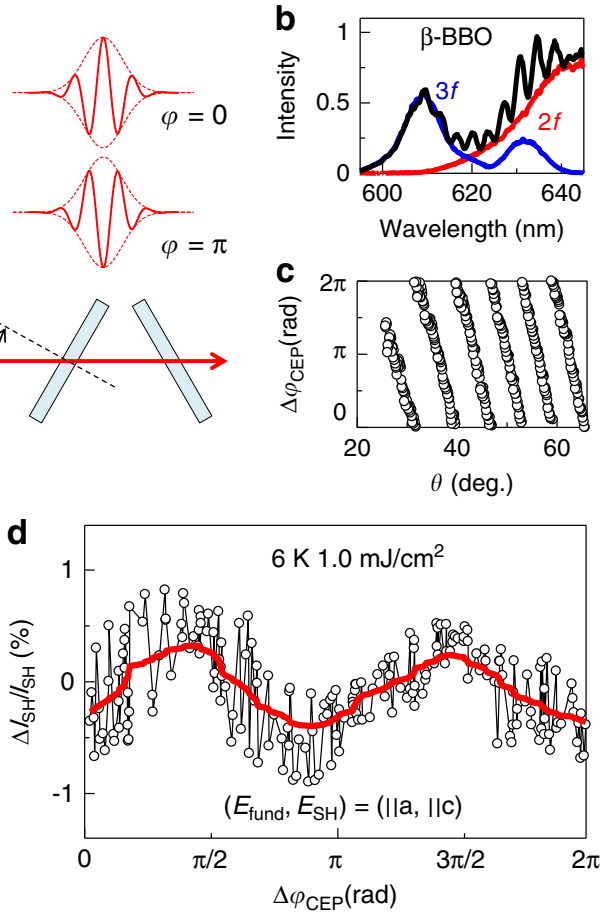

Fig. 3 CEP control of SHG. a Schematic illustrations of the CEP ( $\varphi_{\text {CEP }}=$ $0, \pi$ ) and the pair of BK-7 plates (thickness $1 \mathrm{~mm}$ ) for changing the relative CEP $\Delta \varphi_{\text {CEP }}(\theta$ : incident angle of light). $\mathbf{b}$ Interference spectrum between $2 f$ and $3 f$ of the fundamental light. The $2 f$ and $3 f$ are generated using $\beta$-BBO. c $\Delta \varphi$ as a function of $\theta$ (which is obtained by the $2 f-3 f$ interferometer). d Intensity change of the SHG $\left(\Delta l_{\mathrm{SH}} / I_{\mathrm{SH}}\right)$ as a function of $\Delta \varphi$. The red curve is obtained by averaging the data (guide to the eye). 

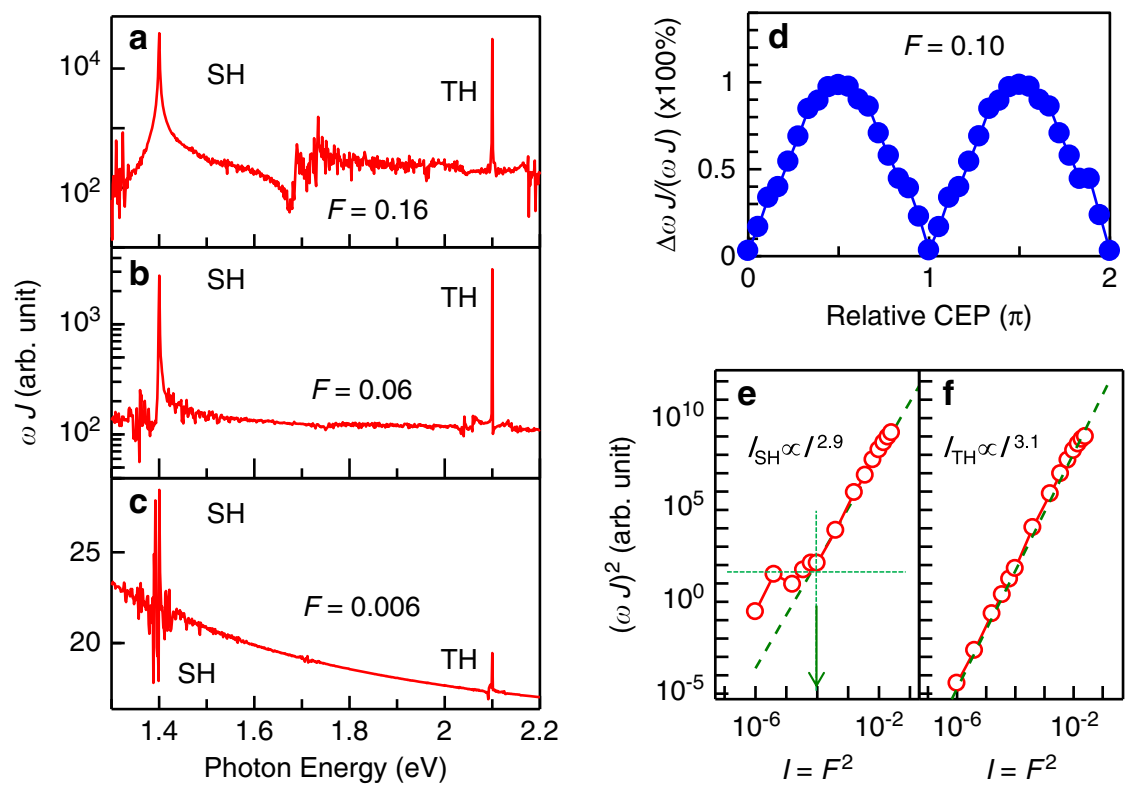

Fig. 4 Calculated spectra of SHG and THG. a-c Calculated spectra of $\omega$ J showing SHG and THG for light-field (which is polarized parallel to the c-axis) $F=0.16 \mathbf{a}, 0.06 \mathbf{b}$, and $0.006 \mathbf{c}$, respectively. $\mathbf{d}$ CEP dependence of the calculated SHG intensity [peak intensities of $\omega J$ at $1.4 \mathrm{eV}(\mathrm{SHG})$ ] for $F=0.1$. e, $\mathbf{f}$ Calculated intensities of SHG e and THG $\mathbf{f}$ as a function of $I=F^{2}$. The green dashed lines in $\mathbf{e}$ and $\mathbf{f}$ indicate $R^{2.9} \mathbf{e}$ and $I^{3.1} \mathbf{f}$.

unconventional SHG is attributed to the non-linear light-induced current.

\section{Discussion}

To clarify the origin of the SHG more in detail, we theoretically calculate the current density $\mathbf{j}$ in a two-dimensional threequarter-filled Hubbard model for a $98 \times 98$-site system in the framework of the time-dependent Hartree-Fock approximation. The details are described in Supplementary note 1, where the emergence of SHG is also checked by the exact diagonalization method for a 16-site system. The calculated SHG and THG spectra $(\omega J, J$ denotes the absolute value of the Fourier transform of $\left.\mathbf{j}^{8,9}\right)$ for $\mathbf{E}_{\mathrm{SH}}|| \mathbf{c}, \mathbf{E}_{\mathrm{TH}}|| \mathbf{c}$ with electric field amplitudes ( $F[\mathrm{~V} /$ $\AA])$ of $0.16,0.06$, and $0.006(\hbar \omega=0.7 \mathrm{eV})$ are, respectively, shown in Fig. $4 \mathrm{a}-\mathrm{c}$. We calculate $\mathbf{j}(t)$ for 500 cycles of the light field. The calculated bandwidth is basically determined by the time window of the Fourier transform in the Hartree-Fock simulation without dephasing. Therefore, we cannot discuss the calculated bandwidth. $I_{\mathrm{SH}}$ is sensitive to the CEP (Fig. 4d), which is consistent with the result shown in Fig. $3 \mathrm{~d}$. On the other hand, the observed anisotropy of the SHG (Fig. 2b) cannot be reproduced by the theory, i.e, $I_{\mathrm{SH}}$ (theory) shows the polarization that is parallel to the fundamental polarization for both $\mathbf{E}_{\text {fund }}|| \mathbf{c}$ and $\mathbf{E}_{\text {fund }}|| \mathbf{a}$. The polarization dependence cannot be understood simply by a point group analysis of $\chi^{(2)}$ tensor for the orthorhombic structure [class $\mathrm{mm} 2\left(\mathrm{C}_{2 \mathrm{v}}\right)$ (after due consideration of the symmetry breaking uniaxially induced by $\mathbf{j}$ )] (Supplementary note 2). However, the results of transient reflectivity measurements do not contradict the above results of the SHG, i.e., the responses of $\mathbf{E}_{\mathrm{pr}}|| \mathbf{c}$ are larger than $\mathbf{E}_{\mathrm{pr}}|| \mathbf{a}$ for both $\mathbf{E}_{\mathrm{pu}}|| \mathbf{a}$ and $\mathbf{E}_{\mathrm{pu}}|| \mathbf{c}\left(\mathbf{E}_{\mathrm{pu}}\right.$ and $\mathbf{E}_{\mathrm{pr}}$ are the electric fields of pump-lights and probe-lights, respectively) (Supplementary note 3 ). This result shows that $\mathbf{E}_{\text {fund }}|| \mathbf{a}$ induces charge motion along the c-axis.

Resistivity anisotropy under hole doping is attributed to the van Hove singularity in the density of states $^{33}$. Under photoirradiation, the situation is similar in the sense that the carriers in the HOMO band is largely transferred into an otherwise empty band through intramolecular charge motion by the strong light field. It is noteworthy that the van Hove singularity, which is located on the $Z$ point, corresponds to the momentum along the c-axis. Because the remaining carriers in the HOMO band would possess momenta mainly along the c-axis, it is natural for charge to oscillate along the $\mathrm{c}$-axis. Considering such anisotropic cooperativity, the polarization dependence is attributed to the van Hove singularity.

As to the calculated excitation intensity ( $I$, defined as $F^{2}$ ) dependences [Fig. 4e (SHG) and Fig. 4f (THG) for electrons in the HOMOs], we notice that $I_{\mathrm{SH}} \propto I^{2.9}$ for $I>10^{-4}$ and $I_{\mathrm{TH}} \propto$ $I^{3.1}$ for $I>10^{-6}$ as shown by the green dashed lines. Above the threshold in the theory (Supplementary note 4 ), $I_{\mathrm{SH}} \propto I^{2.9}$ does not agree with the experimental result $I_{\mathrm{SH}} \propto I^{2.1}$ (inset of Fig. 2a). It would be caused by the fact that the intra-molecular charge motion is not taken into account, though the intra-dimer charge motion is fully taken into account. In fact, the Fourier intensity of the time profile of the charge density in the HOMO of a molecule (to which the intra-molecular optical transition is sensitive) shows a square dependence, which is consistent with our experimental result (Supplementary note 5).

Another important issue is that the spectral bandwidth of the SHG $(130 \mathrm{meV})$ in Fig. $2 \mathrm{a}$ is narrower than that of the 6-fs excitation pulse [almost equal to the Fourier limit of the pulse $(500 \mathrm{meV})]$. The $0.6-2 \mathrm{eV}$ is known as a spectral window in the organic superconductors, i.e., we have no clear reflectivity and absorption bands for | |c polarization. Therefore, a spectral deformation of the SHG $\left(\mathbf{E}_{\mathrm{SH}}|| \mathbf{c}\right)$ owing to an absorption loss is not the reason of the narrow bandwidth (Supplementary note 6). Considering that the band width of the THG $(430 \mathrm{meV})$ is close to that of the 6-fs excitation pulse, the coherence of the SHG survives ca. $30 \mathrm{fs}$ after the light-field application. Note that the wavelength dispersion of the phase-matching condition of SHG for the reflection configuration is as small as that of THG. The narrow bandwidth of the SHG is not attributed to that.

The coherence time of the SHG (30 fs) is comparable to the electronic scattering time of $\sim 40 \mathrm{fs}(=h /(0.1 \mathrm{eV}))$ in organic superconductors, indicating that the coherence time is governed by the electronic scattering (Supplementary note 7). This is consistent with the fact that the bandwidth of the SHG is 
independent of temperature as shown in Fig. 2d. On the other hand, the intensity increases toward $T_{\mathrm{SC}}$, suggesting that such non-linear charge motion on the length scale of a few molecules is enhanced by the superconducting fluctuations. Thus, 6-fs field application on the time scale much shorter than the electron scattering time of ca. $40 \mathrm{fs}$ is an issue very different from the HHG driven by terahertz and mid-infrared fields, where the electric field continues to be applied even after a substantial number of scatterings occur. This is a very important perspective for petahertz functions and attosecond science of superconductors and of strongly correlated systems in addition to already realized terahertz functions of them ${ }^{15,16,26,27}$.

In summary, this article demonstrates SHG in the centrosymmetric organic superconductor $\kappa-(\mathrm{BEDT}-\mathrm{TTF})_{2} \mathrm{Cu}[\mathrm{N}$ $\left.(\mathrm{CN})_{2}\right] \mathrm{Br}$. The SHG is enhanced toward $T_{\mathrm{SC}}$. A narrow bandwidth of $130 \mathrm{meV}$ shows that the coherence of the SHG survives for $30 \mathrm{fs}$ after the $6 \mathrm{fs}$ light-field application. The CEP dependence indicates that the SHG is induced by the non-linear petahertz current.

\section{Methods}

Sample preparation. Single crystals of $\kappa-(\mathrm{BEDT}-\mathrm{TTF})_{2} \mathrm{Cu}\left[\mathrm{N}(\mathrm{CN})_{2}\right] \mathrm{Br}$ were grown under dried nitrogen gas atmosphere by electrochemical oxidation of BEDT-TTF $(80 \mathrm{mg})$ dissolved in $100 \mathrm{ml}$ of $1,1,2$-trichloroethane $(10 \% \mathrm{v} / \mathrm{v}$ ethanol) in the presence of supporting electrolytes $\mathrm{NaN}(\mathrm{CN})_{2}, \mathrm{CuBr}$, and 18crown- 6 ether ${ }^{29}$. A thick plate-like crystal $(0.7 \times 0.5 \times 0.8 \mathrm{~mm}$ for $\mathbf{a}, \mathbf{b}$, and $\mathbf{c}$-axes, respectively) was selected for optical measurements. The a-c crystal plane corresponds to the two-dimensional conductive layer.

6 fs infrared pulse generation. A broadband infrared spectrum covering 1.2-2.3 $\mu \mathrm{m}$ of the $6 \mathrm{fs}$ pulse is obtained by focusing a carrier-envelope phase (CEP) stabilized idler pulse $(1.7 \mu \mathrm{m})$ from an optical parametric amplifier (Quantronix HETOPAS pumped by Spectra-Physics Spitfire-Ace) onto a hollow fiber set within a $\mathrm{Kr}$-filled chamber (Femtolasers). Pulse compression is performed using both active mirror (OKO, 19-ch linear MMDM) and chirped mirror (Femtolasers and SigmaKoki) techniques 29,34

Measurement and CEP control of SHG. We performed SHG and THG measurements for the single crystal using a $6 \mathrm{fs}$ pulse with a reflection geometry (incident angle is smaller than $3^{\circ}$ ). The intensity and polarization of the fundamental (excitation range: $0.01-2 \mathrm{~mJ} / \mathrm{cm}^{2}$ ) pulse are controlled by a pair of wire-grid $\mathrm{CaF}_{2}$ polarizers ${ }^{34}$. The SHG and the THG are detected by a photomultiplier tube (Hamamatsu R13456) after passing through a spectrometer (JASCO, M10). The CEP of the fundamental pulse is controlled by a pair of glass plates with the incidence angle of $\theta$ (Fig. $3 \mathrm{a}$ ) and detected by the $2 f-3 f$ interferometer ( $2 f$ and $3 f$ are generated using $\beta$-BBO) (Fig. $3 b, c)$.

\section{Data availability}

The data that support the plots within this paper and other findings of this study are available from the corresponding author upon reasonable request.

\section{Code availability}

All relevant codes are available from the corresponding author upon reasonable request.

Received: 5 March 2020; Accepted: 20 July 2020;

Published online: 18 August 2020

\section{References}

1. Chin, H. A., Calderón, O. G. \& Kono, J. Extreme midinfrared nonlinear optics in semiconductors. Phys. Rev. Lett. 86, 3292-3295 (2001).

2. Ghimire, S. et al. Observation of high-order harmonic generation in a bulk crystal. Nat. Phys. 7, 138-141 (2011).

3. Luu, T. T. et al. Extreme ultraviolet high-harmonic spectroscopy of solids. Nature 521, 498-502 (2015).

4. Schubert, O. et al. Sub-cycle control of terahertz high-harmonic generation by dynamical Bloch oscillations. Nat. Photon. 8, 119-123 (2014).

5. Yoshikawa, N., Tamaya, T. \& Tanaka, K. High-harmonic generation in graphene enhanced by elliptically polarized light excitation. Science 356, 736-738 (2017).
6. Herink, G. et al. Field-driven photoemission from nanostructures quenches the quiver motion. Nature 483, 190-193 (2012).

7. Pronin, K. A., Bandrauk, A. D. \& Ovchinnikov, A. A. Harmonic generation by a one-dimensional conductor: exact results. Phys. Rev. B 50, 3473-3476(R) (1994).

8. Silva, R. E. F. et al. High-harmonic spectroscopy of ultrafast manybody dynamics in strongly correlated systems. Nat. Photon. 12, 266-270 (2018).

9. Murakami, Y., Eckstein, M. \& Werner, P. High-harmonic generation in Mott insulators. Phys. Rev. Lett. 121, 057405 (2018).

10. Schiffrin, A. et al. Optical-field-induced current in dielectrics. Nature 493, 70-74 (2013).

11. Higuchi, T. et al. Light-field-driven currents in graphene. Nature 550, 224-228 (2017).

12. Piglosiewicz, B. et al. Carrier-envelope phase effects on the strong-field photoemission of electrons from metallic nanostructures. Nat. Photon. 8, 37-42 (2014)

13. Khurgin, J. B. et al. Current induced second harmonic generation in semiconductors. Appl. Phys. Lett. 67, 1113-1115 (1995).

14. Bykov, A. Y. Second harmonic generation in multilayer graphene induced by direct electric current. Phys. Rev. B 85, 12413(R) (2012).

15. Tokman, M. et al. Second harmonic generation in graphene dressed by a strong terahertz field. Phys. Rev. B 99, 155411 (2019).

16. Vaswani, C. et al. Terahertz second harmonic generation from lightwave acceleration of symmetry-breaking nonlinear supercurrents. Phys. Rev. Lett. 124, 207003 (2020).

17. Kagawa, F., Miyagawa, K. \& Kanoda, K. Unconventional critical behavior in a quasi-two-dimensional organic conductor. Nature 436, 534-537 (2005).

18. Sasaki, T., Yoneyama, N., Matsuyama, A. \& Kobayashi, N. Magnetic and electronic phase diagram and superconductivity in the organic superconductors k-(ET) 2 X. Phys. Rev. B 65, 060505(R) (2002).

19. McKenzie, R. H. Similarities between organic and cuprate superconductors. Science 278, 820-821 (1997).

20. Lang, M., Steglich, F., Toyota, N. \& Sasaki, T. Fluctuation effects and mixedstate properties of the layered organic superconductors $\kappa$-(BEDT-TTF $)_{2} \mathrm{Cu}$ $(\mathrm{NCS})_{2}$ and $\kappa-(\mathrm{BEDT}-\mathrm{TTF})_{2} \mathrm{Cu}\left[\mathrm{N}(\mathrm{CN})_{2}\right]$ Br. Phys. Rev. B 49, 15227-15234 (1994).

21. Kobayashi, T., Ihara, Y., Saito, Y. \& Kawamoto, A. Microscopic observation of superconducting fluctuations in $\kappa-(\mathrm{BEDT}-\mathrm{TTF})_{2} \mathrm{Cu}\left[\mathrm{N}(\mathrm{CN})_{2}\right] \mathrm{Br}$ by ${ }^{13} \mathrm{C} \mathrm{NMR}$ spectroscopy. Phys. Rev. B 89, 165141 (2014).

22. Han, S. G. et al. Femtosecond optical detection of quasiparticle dynamics in high-Tc $\mathrm{YBa}_{2} \mathrm{Cu}_{3} \mathrm{O}_{7-\delta}$ superconducting thin films. Phys. Rev. Lett. 65, 2708-2711 (1990).

23. Demsar, J. et al. Superconducting gap $\Delta \mathrm{c}$, the pseudogap $\Delta \mathrm{p}$, and pair fluctuations above $T c$ in overdoped $\mathrm{Y}_{1-x} \mathrm{Ca}_{x} \mathrm{Ba}_{2} \mathrm{Cu}_{3} \mathrm{O}_{7-\delta}$ from femtosecond time-domain spectroscopy. Phys. Rev. Lett. 82, 4918-4921 (1999).

24. Kaindl, R. A. et al. Ultrafast mid-infrared response of $\mathrm{YBa}_{2} \mathrm{Cu}_{3} \mathrm{O}_{7-\delta}$. Science 287, 470-473 (2000).

25. Toda, Y., Mertelj, T., Naito, T. \& Mihailovic, D. Femtosecond carrier relaxation dynamics and photoinduced phase separation in $\kappa$ (BEDT-TTF $)_{2} \mathrm{Cu}\left[\mathrm{N}(\mathrm{CN})_{2}\right] \mathrm{X}(\mathrm{X}=\mathrm{Br}, \mathrm{Cl})$. Phys. Rev. Lett. 107, 227002 (2011).

26. Matsunaga, R. et al. Light-induced collective pseudospin precession resonating with Higgs mode in a superconductor. Science 345, 1145-1149 (2014).

27. Fausti, D. et al. Light-induced superconductivity in a stripe-ordered cuprate Science 331, 189-191 (2011)

28. Mitrano, M. et al. Possible light induced superconductivity in $\mathrm{K}_{3} \mathrm{C}_{60}$ at high temperature. Nature 530, 461-464 (2016)

29. Kawakami, Y. et al. Nonlinear charge oscillation driven by a single-cycle light field in an organic superconductor. Nat. Photon. 12, 474-478 (2018).

30. Mori, T., Mori, H. \& Tanaka, S. Structural genealogy of BEDT-TTF-based organic conductors II. Inclined molecules: $\theta, \alpha$, and $\kappa$ phases. Bull. Chem. Soc. Jpn. 72, 179-197 (1999).

31. Watanabe, $\mathrm{M}$. et al. Low temperature superstructure and transfer integrals in $\kappa-(\mathrm{BEDT}-\mathrm{TTF})_{2} \mathrm{Cu}\left[\mathrm{N}(\mathrm{CN})_{2}\right] \mathrm{X}: \mathrm{X}=\mathrm{Cl}$, Br. Synth. Met. 103, 1909-1910 (1999).

32. Sipe, J. E., Moss, D. J. \& Driel, H. M. Phenomenological theory of optical second- and third-harmonic generation from cubic centrosymmetric crystals. Phys. Rev. B 35, 1129-1141 (1987).

33. Kawasugi, Y. et al. Non-Fermi-liquid behavior and doping asymmetry in an organic Mott insulator interface. Phys. Rev. B 100, 115141 (2019).

34. Kawakami, Y. et al. Polarization selectivity of charge localization induced by a 7-fs nearly single-cycle light field in an organic metal. Phys. Rev. B 95, 201105 (R) (2017).

\section{Acknowledgements}

This work was supported by JST CREST (JPMJCR1901), MEXT Q-LEAP (JPMXS0118067426), JSPS KAKENHI (JP15H02100, JP20K03800, JP17K14317, JP18H01144, JP19H01833, JP19H00891, and JP16K05459, Hattori Hokokai Foundation, The Murata Science Foundation, and Toyota Riken Scholar. 


\section{Author contributions}

Y.K., T.A., H.I. and S.Iw developed the 6-fs light source and carried out the SHG/THG measurements using the 6-fs pulse and, with contributions from K. Ya, H.O., and H.K., analyzed the data. G.K., H.M.Y., Y.N., and H.K. performed the synthesis and the characterization of the thin crystal. T.S. performed the synthesis and the characterization of the single crystal. S. Is and K. Yo made theoretical considerations and calculations. S. Iw devised all the experiments. Y.K., K. Yo, and S. Iw wrote the paper after discussing with all the co-authors.

\section{Competing interests}

The authors declare no competing interests.

\section{Additional information}

Supplementary information is available for this paper at https://doi.org/10.1038/s41467020-17776-3.

Correspondence and requests for materials should be addressed to S.I.

Peer review information Nature Communications thanks Tran Trung Luu and the other, anonymous, reviewer(s) for their contribution to the peer review of this work.
Reprints and permission information is available at http://www.nature.com/reprints

Publisher's note Springer Nature remains neutral with regard to jurisdictional claims in published maps and institutional affiliations.

\section{(c) (i)}

Open Access This article is licensed under a Creative Commons Attribution 4.0 International License, which permits use, sharing, adaptation, distribution and reproduction in any medium or format, as long as you give appropriate credit to the original author(s) and the source, provide a link to the Creative Commons license, and indicate if changes were made. The images or other third party material in this article are included in the article's Creative Commons license, unless indicated otherwise in a credit line to the material. If material is not included in the article's Creative Commons license and your intended use is not permitted by statutory regulation or exceeds the permitted use, you will need to obtain permission directly from the copyright holder. To view a copy of this license, visit http://creativecommons.org/ licenses/by/4.0/.

(C) The Author(s) 2020 